\title{
Trypan blue exclusion assay by flow cytometry
}

\author{
B.A. Avelar-Freitas ${ }^{1,2}$, V.G. Almeida ${ }^{1}$, M.C.X. Pinto ${ }^{3}$, F.A.G. Mourão ${ }^{3}$, A.R. Massensini ${ }^{3}$ \\ O.A. Martins-Filho ${ }^{4}$, E. Rocha-Vieira ${ }^{1}$ and G.E.A. Brito-Melo ${ }^{1}$ \\ ${ }^{1}$ Laboratório de Imunologia, Departamento de Farmácia, Universidade Federal dos Vales do Jequitinhonha e Mucuri \\ and Programa Multicêntrico de Pós-graduação em Ciências Fisiológicas, Diamantina, MG, Brasil \\ ${ }^{2}$ Instituto de Ciência e Tecnologia, Universidade Federal dos Vales do Jequitinhonha e Mucuri, Diamantina, MG, Brasil \\ ${ }^{3}$ Departamento de Fisiologia e Farmacologia, Instituto de Ciências Biológicas, Universidade Federal de Minas Gerais, \\ Belo Horizonte, MG, Brasil \\ ${ }^{4}$ Centro de Pesquisas René Rachou, Fundação Osvaldo Cruz, Belo Horizonte, MG, Brasil
}

\begin{abstract}
Dye exclusion tests are used to determine the number of live and dead cells. These assays are based on the principle that intact plasma membranes in live cells exclude specific dyes, whereas dead cells do not. Although widely used, the trypan blue (TB) exclusion assay has limitations. The dye can be incorporated by live cells after a short exposure time, and personal reliability, related to the expertise of the analyst, can affect the results. We propose an alternative assay for evaluating cell viability that combines the TB exclusion test and the high sensitivity of the flow cytometry technique. Previous studies have demonstrated the ability of TB to emit fluorescence when complexed with proteins. According to our results, TB/bovine serum albumin and TB/cytoplasmic protein complexes emit fluorescence at $660 \mathrm{~nm}$, which is detectable by flow cytometry using a $650-\mathrm{nm}$ low-pass band filter. TB at $0.002 \%$ (w/v) was defined as the optimum concentration for distinguishing unstained living cells from fluorescent dead cells, and fluorescence emission was stable for $30 \mathrm{~min}$ after cell treatment. Although previous studies have shown that TB promotes green fluorescence quenching, TB at $0.002 \%$ did not interfere with green fluorescence in human live T-cells stained with anti-CD3/fluorescein isothiocyanate (FITC) monoclonal antibody. We observed a high correlation between the percentage of propidium iodide $+\mathrm{CD} 3 / \mathrm{FITC}^{+}$and $\mathrm{TB}+\mathrm{CD} 3 / \mathrm{FITC}^{+}$cells, as well as similar doublestained cell profiles in flow cytometry dot-plot graphs. Taken together, the results indicate that a TB exclusion assay by flow cytometry can be employed as an alternative tool for quick and reliable cell viability analysis.
\end{abstract}

Key words: Trypan blue; Fluorescence; Flow cytometry; Cytotoxicity

\section{Introduction}

Cell viability analysis is a useful tool in various experimental procedures, including those for tumor susceptibility, microbiological resistance, and spontaneous cell death after submission to different experimental conditions (1-4). It has been established that cell membrane integrity is a basic criterion for distinguishing dead from live cells (5). Thus, dyes capable of selectively penetrating the cytoplasm of dead cells have been widely used as vital dyes. The trypan blue (TB) method is a very common assay for evaluating cytotoxicity in experimental investigations (6-8) where dead cells absorb TB into the cytoplasm because of loss of membrane selectivity, whereas live cells remain unstained (6). Thus, the relative number of dead and live cells is obtained by optical microscopy by counting the number of stained (dead) and unstained (live) cells using a Neubauer chamber. This conventional TB exclusion assay, when used for a large number of samples, can provide lowprecision results because of the lengthy run time and intensive microscopic examination needed (9).

Previous studies have shown that TB-protein complexes emit fluorescence. As demonstrated by Harrisson et al. (10), TB injected subcutaneously into Japanese quails was adsorbed on exogenous yolk, and the binding sites in oocytes were visualized by fluorescence microscopy. Furthermore, Kumar et al. (11) have suggested that TB is an appropriate dye for detection of arbuscular mycorrhiza spores by confocal fluorescence microscopy. This protocol used an excitation wavelength of $488 \mathrm{~nm}$,

Correspondence: G.E.A. Brito-Melo, Laboratório de Imunologia, Departamento de Farmácia, Universidade Federal dos Vales do Jequitinhonha e Mucuri, Campus JK, Rodovia MGT 367, km 583, 5000 Alto da Jacuba, 39100-000 Diamantina, MG, Brasil. Fax: +5538-3532-1200. E-mail: gbrito1998@yahoo.com

Received September 17, 2013. Accepted January 14, 2014. First published online March 18, 2014. 
with emission starting at $585 \mathrm{~nm}$. Although TB has the ability to emit fluorescence when complexed with proteins, few studies have been conducted to improve the TB exclusion test by making the necessary adaptations for flow cytometry analysis. To date, propidium iodide (PI) staining followed by flow cytometry analysis is one of the most widely established assays for viability evaluation $(12,13)$. Similar to TB, PI has the ability to penetrate into dead cells and to complex with DNA (14-16). We propose a TB exclusion test using flow cytometry as an alternative, cheap, and reliable technique to be used together with phenotype analysis, with no interference with cell viability and no quenching of green fluorescence, as has been previously reported (17).

\section{Material and Methods}

\section{Peripheral blood mononuclear cells (PBMCs)}

A venous blood sample (approximately $10 \mathrm{~mL}$ ) from 7 healthy donors (22 \pm 2.0 years of age) was collected in heparinized tubes $6 \mathrm{~h}$ before the experiment. Blood collection was performed between 7:30 and 8:30 am following these stringent blood-drawing criteria: no reported infection or symptoms of infection for 7 days prior to taking the sample, subject reported adequate sleep (6-9 h), no exercise or alcohol use for $24 \mathrm{~h}$ prior to withdrawal of the blood sample, no topical corticosteroid or aspirin use for the previous $48 \mathrm{~h}$, no systemic antihistamines or corticosteroid use for 1 week prior to obtaining the sample, and no immunizations during the previous 3 weeks. All subjects included in this study gave their informed consent, and the local Ethics Committee approved the study (\#CEP/UFVJM146/10).

The blood and all the reagents used for PBMC isolation were kept at room temperature throughout the purification. PBMCs were isolated by centrifugation using Histopaque ${ }^{\circledR} 1077$ (Sigma-Aldrich, USA), as described by Gazzinelli et al. (18) and adapted as follows. Briefly, the blood was gently added over Histopaque and centrifuged at $520 \mathrm{~g}$. PBMCs were collected from the interphase after Ficoll separation and washed three times with PBS $(0.015$ $\mathrm{M}, \mathrm{pH}$ 7.4). The cell suspensions were then adjusted to $1.0 \times 10^{7}$ cells $/ \mathrm{mL}$.

\section{Determination of the fluorescence emitted by the TB-protein complex}

Evaluation of the fluorescence emitted by the TBprotein complex was performed using a Shimadzu RF5301PC spectrofluorophotometer (USA). Briefly, control solutions containing $0.02 \%$ TB (Sigma-Aldrich) or $10 \%$ bovine serum albumin (BSA; INLAB, Brazil), as well as the test solution $(0.02 \%$ TB and $10 \%$ BSA) were prepared using distilled and deionized water, followed by spectrofluorophotometric analysis. We performed emission and excitation scans using the spectrum measurement mode setting to obtain both excitation and emission spectra. The excitation slit was adjusted to 5 and the emission slit to 20; the spectral analysis was accomplished between 220 and $770 \mathrm{~nm}$. Each analysis was performed in triplicate and collected using the RF-5301PC software. The spectral curve was generated and analyzed by the GraphPad Prism 5.0 software (USA). In a complementary experiment, BSA solutions with concentrations ranging from 1.8 to 10.0 $\mathrm{mg} / \mathrm{mL}$ were added to $0.02 \%$ TB $(\mathrm{w} / \mathrm{v})$ solutions and analyzed by spectrofluorophotometry using fixed excitation and emission wavelengths of 488 and $660 \mathrm{~nm}$, respectively. To confirm the fluorescence emitted by the interaction between TB and cytoplasmic proteins, PBMCs were submitted to simultaneous staining with 4',6-diamidino-2phenylindole (DAPI) and $0.002 \% \mathrm{~TB}(\mathrm{w} / \mathrm{v})$ and analyzed by fluorescence microscopy (Axiolmager.M2, Germany).

TB exclusion test by counting in a Neubauer chamber PBMCs $\left(5.0 \times 10^{5}\right.$ cells) were preincubated in a humidified air environment containing $5 \% \mathrm{CO}_{2}$ at $37^{\circ} \mathrm{C}$ or in a water bath at $50^{\circ} \mathrm{C}$ for $30 \mathrm{~min}$. After preincubation, $1.0 \times 10^{5}$ PBMCs were placed in $5-\mathrm{mL}$ round-bottom tubes and incubated with $190 \mu \mathrm{L} 0.4 \%$ TB solution diluted in PBS to perform the TB exclusion test with counting in a Neubauer chamber.

\section{TB exclusion test using flow cytometry assay}

To evaluate the fluorescence emitted by dead cells stained with TB, $10 \mu \mathrm{L}$ PBMC suspension $\left(1.0 \times 10^{5}\right.$ cells $)$ was submitted to heat $\left(50^{\circ} \mathrm{C}\right)$ pretreatment for $30 \mathrm{~min}$, followed by staining with $190 \mu \mathrm{L}$ TB solutions at 0.002 , $0.004,0.04,0.08$, and $0.4 \%(w / v)$ in PBS. The cell suspensions were incubated in an air-humidified environment containing $5 \% \mathrm{CO}_{2}$ at $37^{\circ} \mathrm{C}$ for $5,10,20$, and $30 \mathrm{~min}$. The dead cell suspensions were treated with TB and were evaluated immediately after the addition of dye to be used as the zero time control. The same protocol was performed without the heat pretreatment step to evaluate the fluorescence emitted by live PBMCs. Cells stained with PI were evaluated by flow cytometry for comparison in all the experiments.

To evaluate whether TB promotes quenching of the green fluorescence as previously reported (17), $100 \mu \mathrm{L}$ heparinized blood samples was incubated with $2 \mu \mathrm{L}$ undiluted FITC-conjugated monoclonal anti-human CD3 antibody (Cat. No. 11-0038-73, Clone UCHT1, eBioscience, USA) in the dark for $30 \mathrm{~min}$ at room temperature. Following the incubation, erythrocytes were lysed with $100 \mu \mathrm{L}$ lysing solution (Optlyse-B, Immunotech, USA) for $5 \mathrm{~min}$, followed by the addition of distilled water for $10 \mathrm{~min}$. The cells were washed twice with $1 \mathrm{~mL}$ PBS containing $0.01 \%$ sodium azide and were then distributed into four new tubes. The cells were incubated with $190 \mu \mathrm{L}$ of either a 0.002 or $0.4 \%$ $(\mathrm{w} / \mathrm{v})$ TB solution in two of the tubes, or with $100 \mu \mathrm{L}$ PBS and $1 \mu \mathrm{L}$ of a $50 \mathrm{mg} / \mathrm{mL}$ PI stock solution (Cat. No. 51-66211E, BD Pharmingen, USA) in the third tube. The leukocytes were added to $190 \mu \mathrm{L}$ PBS in the fourth tube. After $15 \mathrm{~min}$, 
15,000 cells were removed from each tube and analyzed by flow cytometry.

\section{Flow cytometry}

The FACScan [Becton \& Dickinson (BD), USA] flow cytometer used in this study was equipped with a blue argon laser (488 nm) and the following filters: 530/30 nm (FL1 = green fluorescence) and 586/42 (FL2=orange fluorescence) band-pass filters, as well as the 650/LP (FL3 $=$ red fluorescence) long-pass filter. The size and granularity parameters were evaluated with the 488-nm detectors (blue) based on forward and side-scattered light, respectively. For both $\mathrm{PI}$ - and TB-treated cells, 15,000 events were acquired into the lymphocyte gate based on size vs granularity $(F S C \times S S C)$ dot plots. The fluorescence was evaluated in FL3 histograms or FL1 1 FL3 dot plots. Unstained and stained cells were reported as a percentage of live and dead cells, respectively. Calibrite ${ }^{\mathrm{TM}}$ beads (BD, Cat. No. 340486) were used for the three-color flow cytometer compensation setup. The instrument settings for compensation were FL1: 1.3\% FL2; FL2: 20.0\% FL1; FL2: 0.0\% FL3; and FL3: 12.7\% FL2.

\section{Statistical analysis}

GraphPad Prism (version 5.00 for Windows; GraphPad Software, USA, www.graphpad.com) was used for statistical analysis. Data are reported as means $\pm S D$. The ShapiroWilk test was used to assess the normality of the data. The two-way repeated-measures ANOVA was used to compare the viability assays, followed by the Tukey post hoc test when a significant $F$ value was observed. Correlations between the viability assays were assessed by the Pearson correlation test. A significance level of $\mathrm{P} \leq 0.05$ was used.

\section{Results}

\section{TB-protein complex emits fluorescence}

Previous studies have shown that TB emits fluorescence when complexed to proteins (10). We analyzed the excitation and emission spectra using a TB solution at $0.02 \%$ or PBS containing $10 \%$ BSA, as well as a solution containing the TB-BSA complex to determine the optimum wavelengths for excitation and emission to be used in flow cytometry assays. The TB-BSA excitation spectrum presented strong maxima at 296, 485, and $648 \mathrm{~nm}$ (Figure 1A). Maximum emission was observed at 483 and $660 \mathrm{~nm}$ (Figure 1B), the latter being detected by the $650 \mathrm{~nm} / \mathrm{LP}$ (FL3) long-pass filter of the FACScan cytometer. As demonstrated, there were no peaks in the emission spectrum curves corresponding to TB alone and not in the form of a complex with proteins. On the other hand, BSA solution presented an emission maximum at $463 \mathrm{~nm}$ in a region of the spectrum not detectable by the FL3 long-pass filter of the FACScan cytometer. Since the FACScan uses a laser light source at $488 \mathrm{~nm}$, we evaluated the fluorescence emitted by the TB-BSA complex, fixing the excitation and emission wavelengths at 488 and $660 \mathrm{~nm}$, respectively. By maintaining the TB concentration at $0.02 \%$ and by adding BSA solutions ranging from 1.8 to $10 \mathrm{mg} / \mathrm{mL}$, the fluorescence emitted by the TB-BSA complex was observed to be dose-dependent (Figure 2A). Because the TB-BSA complex emits fluorescence, we evaluated whether TB inside cells in the form of complexes with cytoplasmic proteins could present a similar behavior. PBMCs were treated with TB and DAPI, simultaneously. According to the results, the cells containing TB-cytoplasmic protein complexes emitted fluorescence detectable by fluorescence microscopy at the excitation wavelength of $488 \mathrm{~nm}$ and the emission filter of $650 \mathrm{~nm}$ (Figure 2B).

\section{Standardization of the TB exclusion assay using flow cytometry}

Because TB can form complexes with proteins located in the cytoplasm and in the plasma membrane, different TB concentrations, as well as incubation times, were used to determine the optimum experimental condition for
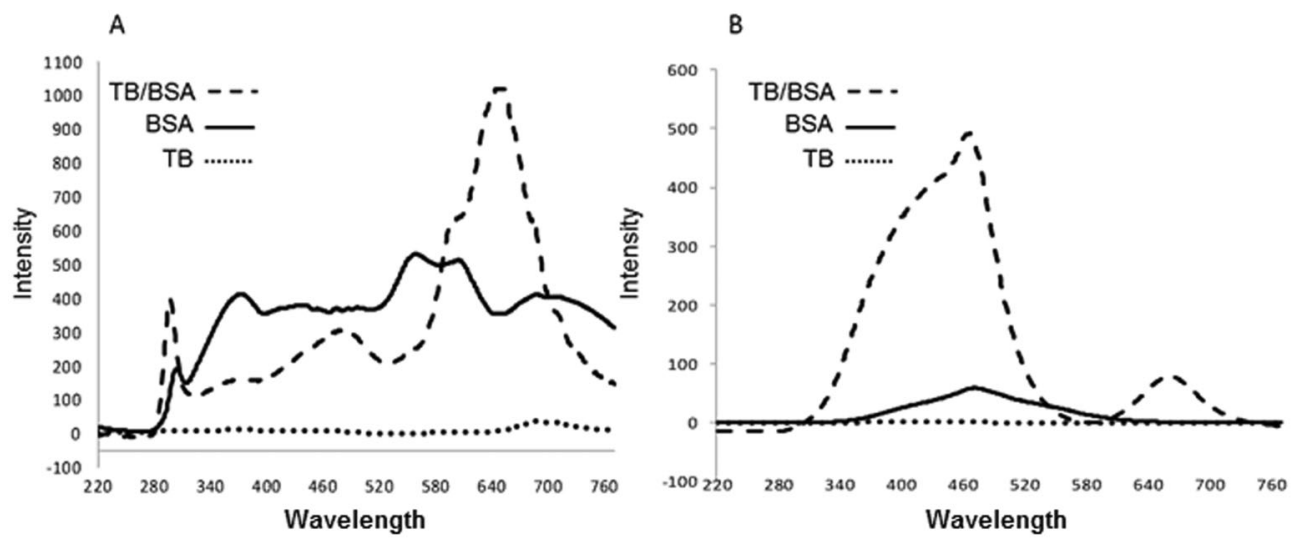

Figure 1. Spectrofluorophotometry analysis of the excitation $(A)$ and emission $(B)$ spectra of the solutions containing $0.02 \%(\mathrm{w} / \mathrm{v})$ trypan blue (TB, dotted line), bovine serum albumin (BSA, solid line) at 10\% (w/v), and TB-BSA (dashed line) interaction. 

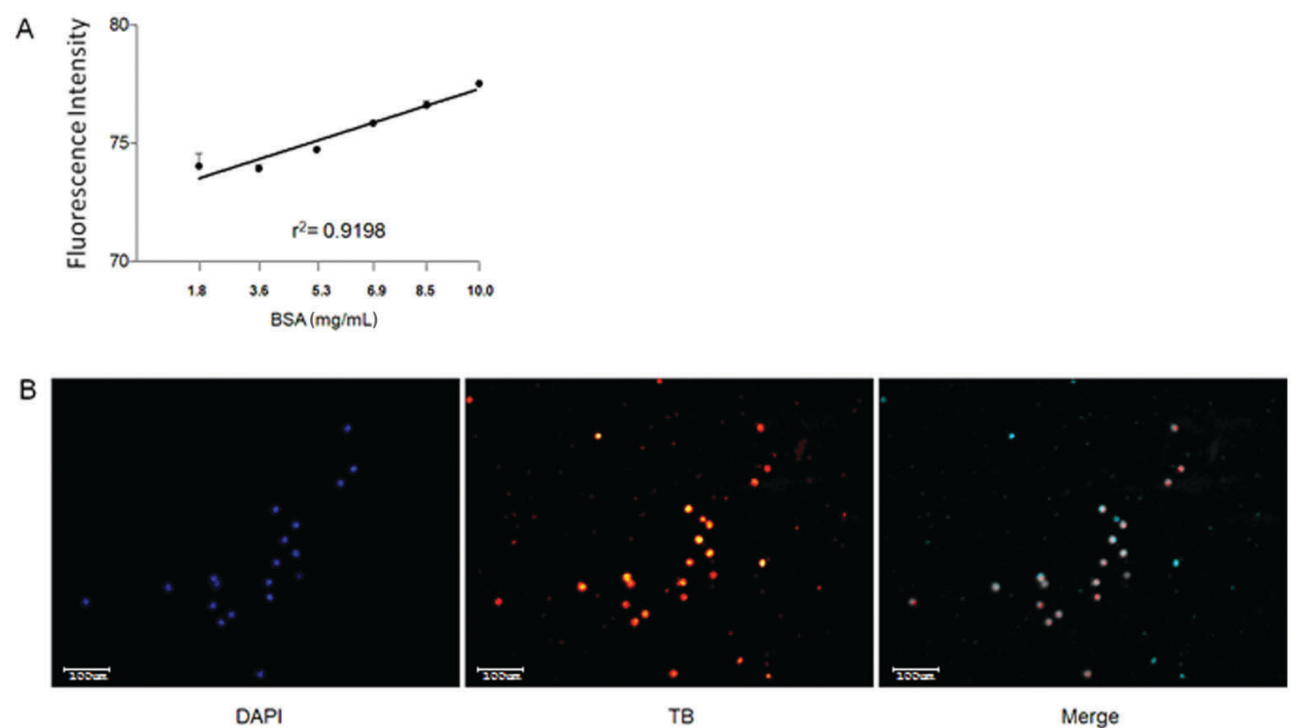

Figure 2. Fluorescence analysis of the trypan blue (TB)-bovine serum albumin (BSA) interaction. $A$, Increasing BSA concentrations were added to $0.002 \%$ TB solution. The fluorimeter was configured to use fixed excitation and emission wavelengths at 488 and $660 \mathrm{~nm}$, respectively. B, Human PBMC co-stained with DAPI and TB. DAPI excitation at $365 \mathrm{~nm}$ and the emission band-pass filter DAPI-445/50, TB excitation at $488 \mathrm{~nm}$ and fluorescence emission detected by LP/515.

distinguishing live cells, whose TB-protein interactions exist only on the cell surface, and dead cells, whose TB-protein complex exists both in the cytoplasm and on the cell surface. A previous study (9) showed that live cells in contact with $0.4 \%$ TB for more than $5 \mathrm{~min}$ may incorporate the dye, leading to overestimation of the number of dead cells. We therefore evaluated whether the optimum TB concentration for use with flow cytometry had a similar imprecision. As shown in Figure 3, 0.002\% (w/v) TB distinguished live and dead cells with the optimal fluorescence emission at $660 \mathrm{~nm}$ (FL3), and was able to distinguish dead (stained) cells, keeping live (unstained) cells in a negative region usually located between $10^{\circ}$ and $10^{1}$ on FL3-based histograms. Additionally, $0.002 \%(\mathrm{w} / \mathrm{v})$ TB did not change the fluorescence intensity emitted by live cells that were incubated for 30 min after addition of the dye (Figure 4). All the results were comparable to those of the PI assay.

\section{Comparison between the TB exclusion test by flow cytometry, PI staining, and the conventional TB exclusion test by cell counting in the Neubauer chamber}

A comparison between the TB exclusion assay by flow cytometry $[0.002 \%(w / v) ~ T B]$, the PI exclusion test by flow cytometry, and the percentage of cells stained with $0.4 \%$ TB counted in a Neubauer chamber was performed to verify whether the results obtained with the standardized cytometry-based TB assay were similar to those obtained by conventional methods. The cell counting in a Neubauer chamber was performed no later than 3 min after cell staining. The three methods presented no differences in the percentage of dead or live cells in both protocols (with or without heat pretreatment; Figure $5 \mathrm{~A}$ ). There was a very high correlation between the TB exclusion assay by flow cytometry, the $\mathrm{PI}\left(r^{2}=0.9758\right)$ assay, and the Neubauer chamber $\left(r^{2}=0.8986\right)$ assay (Figure $\left.5 \mathrm{~B}\right)$. Interestingly, the assays using $\mathrm{PI}$ and TB analyzed on the long-pass filter at FL3 $=650 \mathrm{~nm} / \mathrm{LP}$ had very similar profiles in the FL3fluorescence histogram analysis (Figure $5 C$ ), as well as the dot-plot graphs (data not shown). Furthermore, according to data shown in Figure 1B, fluorescence emitted by TB was detected in the FL3 channel $(650 \mathrm{~nm} / \mathrm{LP})$ on the FACScan cytometer and, contrary to PI, TB-protein fluorescence was not detected in the FL2 channel (Figure 5D).

\section{TB at $\mathbf{0 . 0 0 2} \%(\mathbf{w} / \mathbf{v})$ does not promote quenching in FL1}

TB at 0.2 and $0.4 \%$ promoted a decrease in FL1 fluorescence (quenching) under acidic conditions. To determine whether cell treatment with $0.002 \%$ TB at $\mathrm{pH} 7.2$ promoted FL1 quenching, we stained $100 \mu \mathrm{L}$ peripheral blood with specific monoclonal antibody antihuman CD3-FITC followed by TB or PI staining. The results showed that $0.002 \%(\mathrm{w} / \mathrm{v}) \mathrm{TB}$, at physiological $\mathrm{pH}$, did not interfere with FL1-530/30 nm fluorescence (Figure 6) and produced results similar to those obtained by the cell cultures treated with $\mathrm{PI}$ or PBS (control). After cell death was induced using heat $\left(50^{\circ} \mathrm{C}\right)$ pretreatment, the flow cytometry exclusion assays using TB or PI presented a similar percentage of live and dead cells, confirmed by the high correlation between these methods $\left(r^{2}=0.9805\right)$, as well as very similar dot-plot profiles in flow cytometry analysis (Figure 7). 
Live PBMC

PI
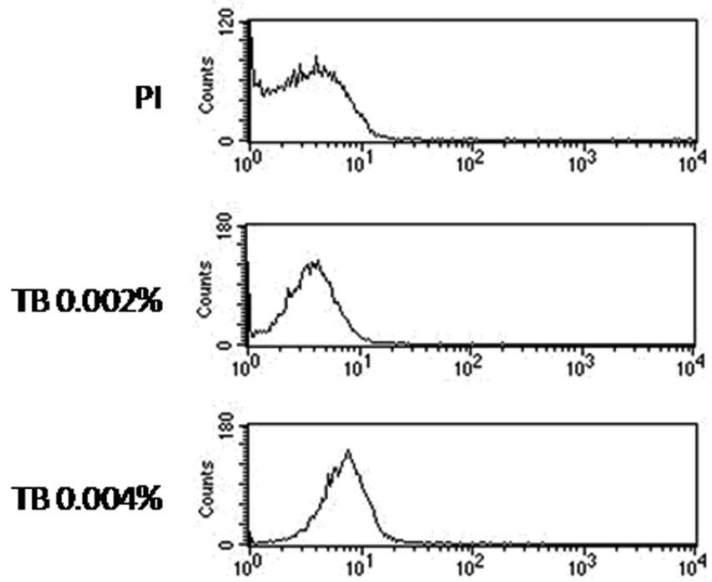

IB 0.04\%

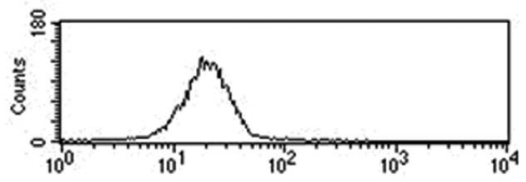

TB 0.08\%

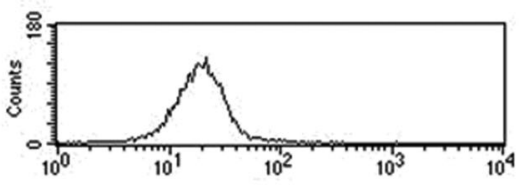

TB 0.4\%

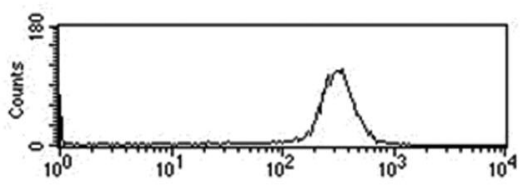

\section{DeadPBMC}
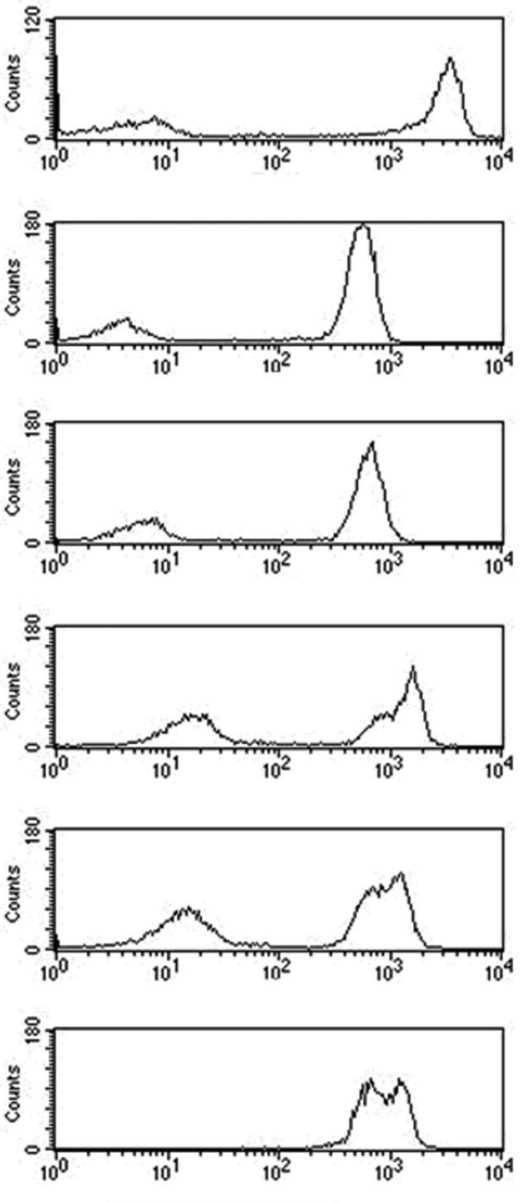

$660 \mathrm{~nm} / \mathrm{P}(\mathrm{F} L 3)$

Figure 3. Evaluation of the optimum concentration for trypan blue (TB) solution ranging from 0.002 to $0.4 \%$ to discriminate between live and dead peripheral blood mononuclear cells (PBMC). The analysis was performed on a FACScan cytometer (Becton \& Dickinson, USA) using the long-pass filter 650/LP (FL3 detector). Staining with propidium iodide (PI) was used as a reference.

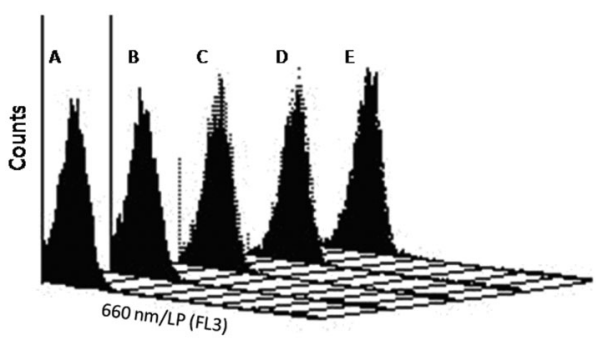

Figure 4. Histogram analysis of the trypan blue (TB) exclusion assay by flow cytometry to evaluate TB (at $0.002 \%$, w/v) incorporation by live cells $0(A), 5(B), 10(C), 20(D)$, and 30 $(E)$ min after dye addition.

\section{Discussion}

The number of live and dead cells in an experiment can be estimated by the use of several markers including dyes that intercalate into DNA (e.g., ethidium monoazide, $\mathrm{PI}, 7$-aminoactinomycin D, DAPI), reagents that bind to phosphatidylserine (Annexin V), dyes that stain cells that lose membrane selectivity, and amine reactive dyes (UViD, ViViD, GrViD, OrViD: UV, violet, green, and orange fluorescence, respectively). Among the tests using dyes that penetrate into cells that lose membrane selectivity or that intercalate with DNA, the TB exclusion assay, using optical microscopy, and the labeling of cells with $\mathrm{PI}$, followed by flow cytometry analysis, are widely used by the scientific community $(14,19)$.

Studies have demonstrated the fluorescence emitted by TB-protein complexes $(10,11,20)$. Davis and Sauter 
A

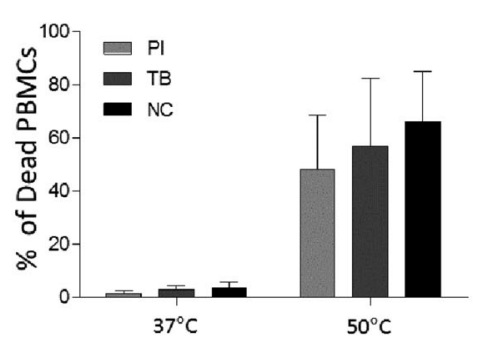

B

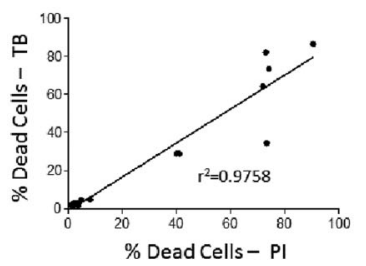

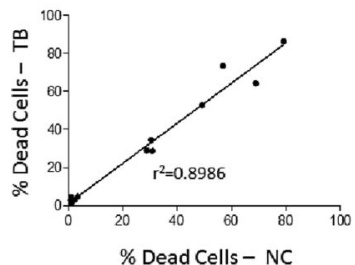

C

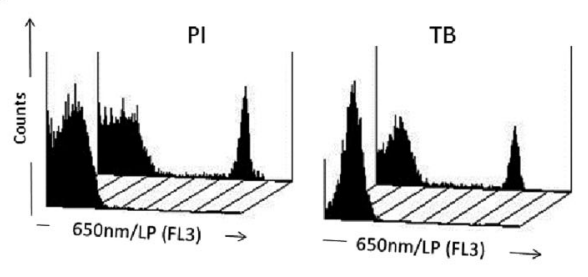

$\mathrm{PI}$
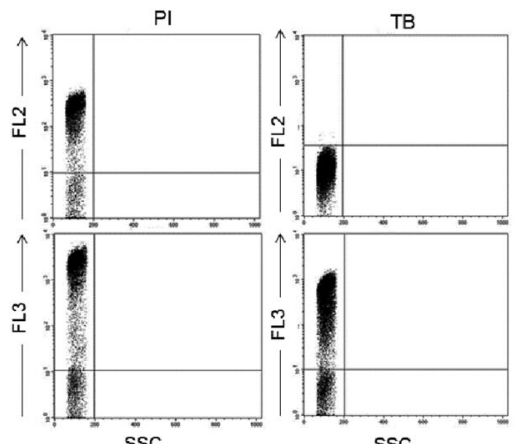

Figure 5. $A$, Comparison of the trypan blue (TB) exclusion test using flow cytometry, propidium iodide (PI) staining and the conventional trypan blue exclusion test employing cell counting in a Neubauer chamber (NC). B, Pearson's correlation test between PI and TB or NC and TB. $C$, Fluorescence intensity histogram profiles of the PI and TB flow cytometry analysis. $D$, Fluorescence emitted by cells stained with $\mathrm{PI}$ and TB dyes and analyzed on FL2 (585/42) and FL3 (650 nm/LP) detectors.
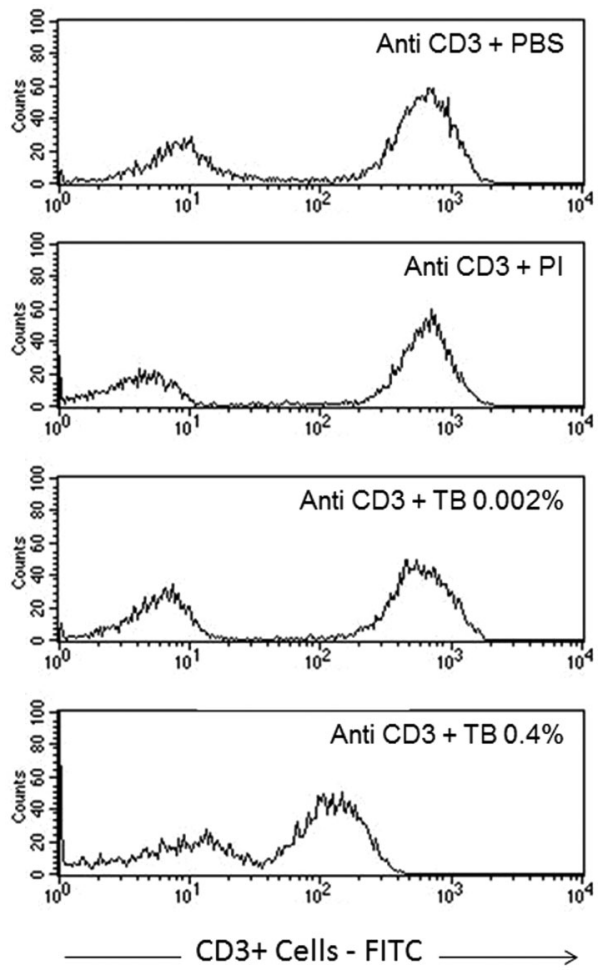

Figure 6. Profile of T-lymphocytes stained with monoclonal antibody anti-CD3-FITC followed by treatment with propidium iodide (PI) and trypan blue (TB) at 0.002 and $0.4 \%(\mathrm{w} / \mathrm{v})$ or PBS (untreated control).
(21) developed a method for detection and characterization of intraembryonic dyes. Using this method, the fluorescence produced by protein-bound TB can be distinguished from non-protein-bound dye. Busetto et al. (22) reported a TB fluorescence-based assay to evaluate the phagocytic action of neutrophils in vitro. In this assay, fixed Candida albicans stained with carboxyfluorescein diacetate succinimidyl ester (CFSE) were added to neutrophil cultures together with TB labeling. Since TB did not penetrate live cells, ingested yeasts retained their green fluorescence, while membrane-bound $C$. albicans displayed a double-positive red and green fluorescence. Because these and other previous studies showed the ability of the TB-protein complex to emit fluorescence, we questioned whether the cells assayed by TB exclusion might also be evaluated by flow cytometry, an issue that had not yet been reported. The main objective of this study was to present an adaptation of a technique already known and widely used in scientific experimentation to be employed as an additional and alternative tool for providing reliable results.

We demonstrated that the TB-BSA interaction emitted fluorescence at 490 and $660 \mathrm{~nm}$. Moreover, human PBMC fluorescence was observed by the overlapping of blue and red fluorescence after simultaneous labeling with DAPI and TB (Figure 2B). This result, along with flow cytometry analysis for cell size (FSC) and granularity (SSC), demonstrated that the red fluorescence evaluated by flow cytometry was not produced by possible technical artifacts. The fluorescence emitted by interactions between TB and 
A

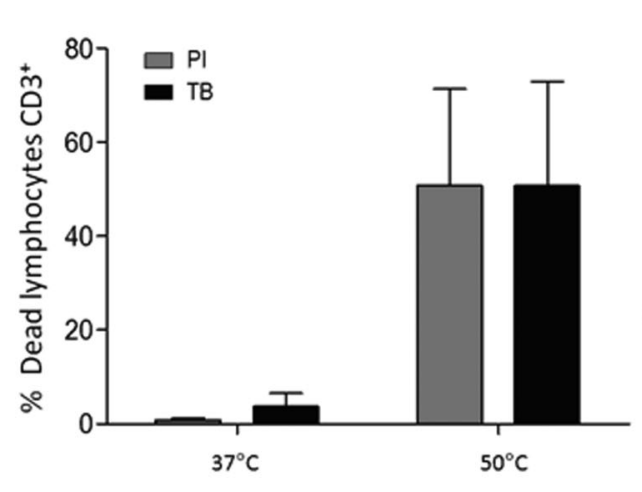

B

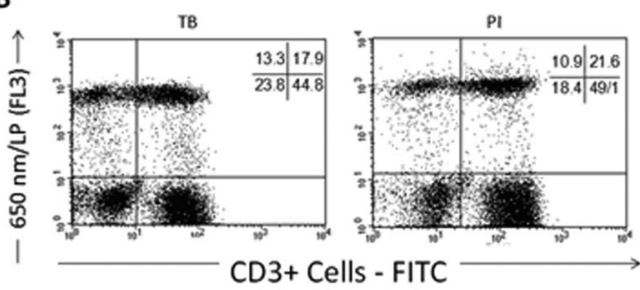

C

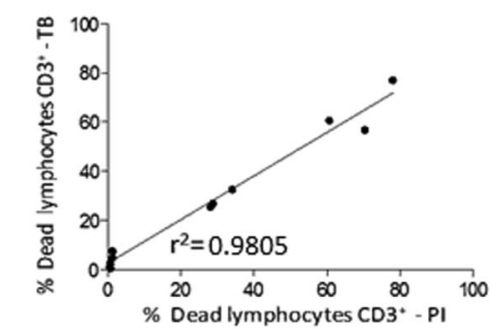

Figure 7. $A$, Percentage of dead human T-lymphocytes (CD3-FITC ${ }^{+}$cells) submitted to cell culture at temperatures $(\mathrm{T})$ of $37^{\circ} \mathrm{C}$ (physiologic temperature) or $50^{\circ} \mathrm{C}$ (high-stress temperature) followed by staining with trypan blue (TB) or propidium iodide (PI). $B$, Dotplot graph profile between human lymphocytes submitted to pretreatment with high-stress temperature $\left(50^{\circ} \mathrm{C}\right)$ followed by staining with TB and PI and monoclonal antibody anti-CD3-FITC ${ }^{+}$. C, Pearson's correlation test between dead $\mathrm{CD}^{+}$lymphocytes using PI and TB flow cytometry assays.

cytoplasmic proteins may, however, be influenced by the diversity and quantity of proteins within specific cell populations. Certain cells, such as monocytes and neutrophils, possess high proteogenic activity to support the effector functions in the phagocytosis process. Large numbers of protein granules inside the cells may lead to the phenomenon known as autofluorescence, a common effect observed in phenotypic analysis by flow cytometry (23). Therefore, it is recommended that, for each cell type, an optimum TB concentration adjustment is required.

Concerning the affinity of TB for proteins, the presence of red fluorescent cells after treatment with TB dye could indicate the presence of the TB-protein complex both inside and outside the cells. Flow cytometry is a highly sensitive technique. Therefore, it was necessary to determine the optimum concentration of TB necessary to distinguish live cells, which have a basal level of fluorescence due to the TB-protein complex on the cell surface from that of dead cells, where the TB-protein complex occurs both in the cytoplasm and outside the cell membrane. Thus, we treated a suspension of live and dead cells using different concentrations of TB to confine the population of live cells, which do not incorporate the dye, into a log-based scale region between $10^{\circ}$ and $10^{1}$ (negative red-fluorescent region) as well as to distinguish dead intracellularly stained cells in FL3-based histograms. Heat pretreatment to induce cell death was chosen to avoid possible chemical interference of the cytotoxic agent with the formation of the TB-protein complex. According to our data, 0.002\% TB promoted results similar to those of PI staining and was defined as the optimal concentration for further analysis (Figure 3). It is important to consider that, in flow cytometry, it is common to use washing solutions supplemented with BSA or other Fc blockers, such as normal serum, to saturate $F c$ receptors on the cell surface. Because TB interacts nonspecifically with proteins, the formation of $\mathrm{TB} / \mathrm{Fc}$ blocker complexes in the extracellular medium is possible. In the assay proposed herein, $0.002 \%$ TB is sufficient to form complexes with soluble proteins, as well as proteins located on the membrane and in the cytoplasm of live and dead cells, respectively. In the data obtained by flow cytometry, all those TB-protein complexes were considered, and the total fluorescence represents the sum of the TB-protein complexes both inside and outside the cells. TB-protein complexes not associated with cells are discarded in the cell population selection step (gated cells). Therefore, the presence of Fc blockers such as BSA in the medium does not interfere with the analysis of live and dead cells. Moreover, our data showed that the use of the washing solution (PBS) containing $0.5 \%$ BSA in the TB exclusion assay by flow cytometry did not cause a change in the cell viability analysis when compared to the protocol where BSA-free PBS was used (data not shown). In addition, the cell surface staining using anti-CD3-FITC monoclonal antibodies was also not affected, showing that $0.002 \%$ TB did not interfere in the quenching pattern presented in this study, even in the presence of Fc blockers. However, in experiments where cell cultures are treated with specific cytotoxic agents, it is recommended that a preliminary experiment be conducted to evaluate the possible chemical interference of the cytotoxic agent with TB-protein complex formation, as well as to evaluate the ability of the compound to emit fluorescence, such as doxorubicin, a widely used drug in cytotoxic assays that 
emits FL3-detectable fluorescence by itself (24).

Previous studies have shown that live cells, when treated with $0.4 \%(\mathrm{w} / \mathrm{v}) \mathrm{TB}$, incorporate the dye after 5 min of TB treatment $(25,26)$. We observed that live human leukocytes treated with $0.002 \%$ TB for 30 min after staining presented no differences in the fluorescence profile (Figure 4). This stable interaction between TB and cytoplasmic proteins could be useful for experiments that require the analysis of a large number of samples. The TB exclusion test by flow cytometry presented a strong positive correlation with the $\mathrm{PI}$ assay and the counting of stained cells in a Neubauer chamber by optical microscopy and confirmed the reliability of the proposed test (Figure 5B). The FL3 histograms obtained from PI and TB fluorescence analysis also presented a very similar pattern (Figure $5 \mathrm{C}$ ). Considering that the FL2 detector is not sensitive to the fluorescence emitted by the TB-BSA complex, the TB exclusion assay by flow cytometry can be performed together with phycoerythrinconjugated antibody staining. This issue represents a technical advantage over the use of $\mathrm{PI}$, since this DNA marker is read simultaneously by FL2 and FL3 detectors (Figure 5D).

According to Van Amersfoort and Van Strijp (27), the use of TB at concentrations higher than $0.1 \%$ under acidic conditions can cause quenching of green fluorescence. The quenching effect promoted by TB on green fluorescence is an undesired event in cell phenotype analysis because this effect could be a limitation to double-staining with FITC-conjugated monoclonal antibodies. Thus, another option was to determine the possibility of simultaneous evaluation of cell viability by TB and cell surface staining using FITC by flow cytometry. PBS was used with the $\mathrm{pH}$ close to 7.2, and the levels of fluorescence emitted by $\mathrm{CD}^{-}-\mathrm{FITC}^{+}$cells were maintained even at high TB

\section{References}

1. Chan LL, Wilkinson AR, Paradis BD, Lai N. Rapid imagebased cytometry for comparison of fluorescent viability staining methods. J Fluoresc 2012; 22: 1301-1311, doi: 10.1007/s10895-012-1072-y.

2. Chen SF, Lu WF, Wen ZY, Li Q, Chen JH. Preparation, characterization and anticancer activity of norcantharidinloaded poly(ethylene glycol)-poly(caprolactone) amphiphilic block copolymer micelles. Pharmazie 2012; 67: 781-788.

3. Altiok N, Ersoz M, Koyuturk M. Estradiol induces JNKdependent apoptosis in glioblastoma cells. Oncol Lett 2011; 2: 1281-1285

4. Puoci F, Morelli C, Cirillo G, Curcio M, Parisi OI, Maris P, et al. Anticancer activity of a quercetin-based polymer towards HeLa cancer cells. Anticancer Res 2012; 32: 28432847.

5. Kroemer G, Galluzzi L, Vandenabeele P, Abrams J, Alnemri $E S$, Baehrecke $E H$, et al. Classification of cell death: recommendations of the Nomenclature Committee on Cell Death 2009. Cell Death Differ 2009; 16: 3-11, doi: 10.1038/ concentrations $(0.4 \%)$. The FL1 fluorescence peak of the cells treated with anti-CD3-FITC and PBS was identical to the fluorescence peak of the cells treated with $0.002 \%$ TB. In comparison with $\mathrm{PI}$ and TB assays after FITC staining, a high correlation was observed, as well as a very similar profile in the dot plot obtained by the two techniques (Figure 7C).

In conclusion, the results are quite consistent and comparable to those obtained by the PI staining method. The large number of cells evaluated ( 30,000 cells), as well as the short time required by the cytometry assay, provide reliable results regarding the real amount of dead and live cells on the samples. Furthermore, the TB exclusion assay by flow cytometry means that simultaneous cell staining with monoclonal antibodies conjugated with fluorochromes detectable on the FL2 (585/42 nm) or FL1 $(530 / 30 \mathrm{~nm})$ channel is possible. This possibility represents an advantage over the $\mathrm{PI}$ assay. Flow cytometry is an expensive technique, but it has been widely employed. Thus, for the laboratories that make use of such equipment in their investigations, the TB exclusion assay by flow cytometry can be a reliable tool for viability analysis and has advantages over traditionally used methods.

\section{Acknowledgments}

We are grateful to Dr. David Lee Nelson for technical review of the manuscript, and to the Brazilian funding agencies FAPEMIG (\#CBB-APQ-04243-10 and \#CBBAPQ-00581-11), CNPq, and CAPES for financial support, as well as to the Multicentric Post-Graduate Program in Physiological Science (SBFIS/UFVJM) and PostGraduate Program in Pharmaceutical Science of the UFVJM. cdd.2008.150.

6. Tennant JR. Evaluation of the trypan blue technique for determination of cell viability. Transplantation 1964; 2 : 685694, doi: 10.1097/00007890-196411000-00001.

7. Song K, Li W, Wang H, Wang H, Liu T, Ning R, et al. Investigation of coculture of human adipose-derived stem cells and mature adipocytes. Appl Biochem Biotechnol 2012; 167: 2381-2387, doi: 10.1007/s12010-012-9764-y.

8. Zanatta G, Steffens D, Braghirolli DI, Fernandes RA, Netto CA, Pranke P. Viability of mesenchymal stem cells during electrospinning. Braz J Med Biol Res 2012; 45: 125-130, doi: 10.1590/S0100-879X2011007500163.

9. Kim JS, Nam MH, An SS, Lim CS, Hur DS, Chung C, et al. Comparison of the automated fluorescence microscopic viability test with the conventional and flow cytometry methods. J Clin Lab Anal 2011; 25: 90-94, doi: 10.1002/ jcla.20438.

10. Harrisson F, Callebaut M, Vakaet L. Microspectrographic analysis of trypan blue-induced fluorescence in oocytes of 
the Japanese quail. Histochemistry 1981; 72: 563-578, doi: 10.1007/BF00493276.

11. Kumar T, Majumdar A, Das P, Sarafis V, Ghose M. Trypan blue as a fluorochrome for confocal laser scanning microscopy of arbuscular mycorrhizae in three mangroves. Biotech Histochem 2008; 83: 153-159, doi: 10.1080/ 10520290802336161.

12. Tabera S, Perez-Simon JA, Díez-Campelo M, SanchezAbarca LI, Blanco B, Lopez A, et al. The effect of mesenchymal stem cells on the viability, proliferation and differentiation of B-lymphocytes. Haematologica 2008; 93: 1301-1309, doi: 10.3324/haematol.12857.

13. Edidin M. A rapid, quantitative fluorescence assay for cell damage by cytotoxic antibodies. J Immunol 1970; 104: 1303-1306.

14. Darzynkiewicz Z, Bruno S, Del Bino G, Gorczyca W, Hotz MA, Lassota $P$, et al. Features of apoptotic cells measured by flow cytometry. Cytometry 1992; 13: 795-808, doi: 10.1002/cyto.990130802.

15. Riccardi C, Nicoletti I. Analysis of apoptosis by propidium iodide staining and flow cytometry. Nat Protoc 2006; 1: 1458-1461, doi: 10.1038/nprot.2006.238.

16. Fried J, Perez AG, Clarkson BD. Flow cytofluorometric analysis of cell cycle distributions using propidium iodide. Properties of the method and mathematical analysis of the data. J Cell Biol 1976; 71: 172-181, doi: 10.1083/jcb.71.1. 172.

17. Miliukene VV, Biziuliavichene GI, Khaustova LP, Pilinkene $\mathrm{AV}$, Biziuliavichius GA. [Determination of quantitative parameters of Escherichia coli phagocytosis by mouse peritoneal macrophages]. Tsitologiia 2007; 49: 853-857.

18. Gazzinelli G, Katz N, Rocha RS, Colley DG. Immune responses during human schistosomiasis mansoni. X. Production and standardization of an antigen-induced mitogenic activity by peripheral blood mononuclear cells from treated, but not active cases of schistosomiasis. $J$ Immunol 1983; 130: 2891-2895.

19. Chandra RV, Bhat KM, Jagetia GC. Trypan blue exclusion principle in the evaluation of fibroblast attachment in vitro using V79 cells on the conditioned root surface. Quintessence
Int 2005; 36: 702-706

20. Pereira de Souza CC, Moreira Prado G, da Conceição Freitas RC, Santos Guimarães PS, Calegario de Oliveira L, Brito-Melo GEA, et al. Analysis of Aspergillus nidulans germination, initial growth and carbon source response by flow cytometry. J Basic Microbiol 2011; 51: 459-466, doi: 10.1002/jobm.201000242.

21. Davis HW, Sauter RW. Fluorescence of Trypan blue in frozen-dried embryos of the rat. Histochemistry 1977; 54: 177-189, doi: 10.1007/BF00492240.

22. Busetto S, Trevisan E, Patriarca P, Menegazzi R. A singlestep, sensitive flow cytofluorometric assay for the simultaneous assessment of membrane-bound and ingested Candida albicans in phagocytosing neutrophils. Cytometry A 2004; 58: 201-206, doi: 10.1002/cyto.a.20014.

23. Dorward DA, Lucas CD, Alessandri AL, Marwick JA, Rossi F, Dransfield I, et al. Technical advance: autofluorescencebased sorting: rapid and nonperturbing isolation of ultrapure neutrophils to determine cytokine production. J Leukoc Biol 2013; 94: 193-202, doi: 10.1189/jlb.0113040.

24. Quan ZW, Yang Y, Li JY, Gong W, Qin YY, Li SG. The mechanisms of somatostatin induced enhanced chemosensitivity of gallbladder cancer cell line to doxorubicin: cell cycle modulation plus target enzyme up-regulation. Biomed Pharmacother 2010; 64: 451-457, doi: 10.1016/j.biopha. 2010.01.010

25. Tsaousis KT, Kopsachilis N, Tsinopoulos IT, Dimitrakos SA, Kruse FE, Welge-Luessen U. Time-dependent morphological alterations and viability of cultured human trabecular cells after exposure to Trypan blue. Clin Experiment Ophthalmol 2013; 41: 484-490, doi: 10.1111/ceo.12018.

26. Jones KH, Senft JA. An improved method to determine cell viability by simultaneous staining with fluorescein diacetatepropidium iodide. J Histochem Cytochem 1985; 33: 77-79, doi: 10.1177/33.1.2578146.

27. Van Amersfoort ES, Van Strijp JA. Evaluation of a flow cytometric fluorescence quenching assay of phagocytosis of sensitized sheep erythrocytes by polymorphonuclear leukocytes. Cytometry 1994; 17: 294-301, doi: 10.1002/ cyto. 990170404 